\title{
IMAGINAIRE, VIE ET HYSTERIE CHEZ MERLEAU-PONTY*
}

\author{
Silvana de Souza Ramos** \\ ramos_si@hotmail.com
}

RESUME Dans cet article, j'aborde la perspective idéaliste de la "Phénoménologie de la perception » pour montrer que, dans cet ouvrage, l'expérience du corps propre est décrite à partir de la vision du cogito. Pour expliciter cet idéalisme, j'analyse la principale voix donnée au corps au début de la pensée de Merleau-Ponty: la voix pathologique. Les troubles de Schneider rendent explicite son impuissance symbolique, et cette impuissance est vraiment l'impuissance du corps. Le sujet malade est un être soumis à la normativité immédiate de la vie, raison par laquelle il peut découvrir à chaque moment la finitude et la fragilité de l'existence. J'évoque par contre les notes du cours au Collège de France, de 1954-1955, sur la passivité et les cours sur la Nature, de 1957-1958, lorsque Merleau-Ponty examine la vie et la naissance du sens à partir d'une perspective tout à fait différente. J'analyse aussi la lecture qu'il a faite du «cas Dora » pour montrer que le philosophe trouve dans l'hystérie une expérience symbolique très particulière. Cette expérience exige la compréhension de la puissance symbolique du corps propre, l'abandon de la position idéaliste et la découverte du pouvoir de l'hystérique de narrer l'expérience de l'être.

Mots clés Merleau-Ponty, idéalisme, corps, imagination, pathologie, hystérie.

ABSTRACT In this article, I approach the idealistic perspective of the "Phenomenology of Perception" to show that in that work the experience of the body is described from a vision of the cogito. To explain this idealism, I asses the

** USP - Universidade de São Paulo, São Paulo, SP, Brasil. 
main voice given to the body in the beginning of the thought of Merleau-Ponty: the pathological voice. Schneider's disturbances make explicit its symbolic powerlessness, and that powerlessness is in fact the body's powerlessness. The sick is a being submitted to the immediate normativity of life, reason by which he can find out at every moment the finitude and fragility of existence. On the other hand, I evoke the course notes from Collège de France, of 19541955, about passivity, and the courses about Nature, of 1957-1958, when Merleau-Ponty analyses life and the birth in the sense from an entirely different perspective. Also, I analyze the reading of what he to "case Dora" to show that the philosopher finds in hysteria a very particular symbolic experience. This experience requires understanding the symbolic power of the body itself, the abandonment of the idealist position and the discovery of the power of the hysteric of narrating the experience of being.

Keywords Merleau-Ponty, idealism, body, imagination, pathology, hysteria.

\section{1}

Comment une philosophie de la perception peut-elle être idéaliste? Le corps ne joue-t-il pas ici le rôle principal? Dans le cas de Merleau-Ponty, le problème central est que la description de la perception est construite à partir de la perspective de la conscience. Par conséquent, le mouvement et toutes les expressions du corps sont soumis aux formalisations de ce point de vue. Donc, même s'il dit que la " Phénoménologie de la perception » est profondément ancrée sur l'expérience sensible, le philosophe n'abandonne pas la possibilité d'organiser cette expérience par la perspective du cogito. C'est vrai que MerleauPonty trouve un cogito tacite, car le philosophe montre que le centre de la conscience polarise le spirituel et le physique. À tel point qu'au niveau de la description de l'expérience sensible et de ses dédoublements dans le langage, il n'y a pas de séparation entre le corps et l'âme. Mais il faut se demander qui réalise cette union. Selon Merleau-Ponty, il y a un œil, une vision présente ou actuelle qui concentre notre existence personnelle. Cette conscience recueille la dispersion de l'expérience anonyme et l'ordonne. Cela signifie que l'expérience corporelle a besoin d'un esprit qui lui donne une forme précise, c'est-à-dire, la forme humaine d'accéder au monde perçu et même à la dimension abstraite de l'expérience ${ }^{1 .}$

1 « Si justement il doit y avoir conscience, si quelque chose doit apparaître à quelqu'un, il est nécessaire que derrière toutes nos pensées particulières se creuse un réduit de non-être, un Soi » (Merleau-Ponty, 1945, p. 
La description du monde de la perception chez Merleau-Ponty n'est pas simplement le fruit d'une spéculation philosophique. Elle est basée sur les données de la psychologie et de la biologie. Merleau-Ponty utilise les études des cas pathologiques pour découvrir l'expérience anonyme qui se déroule en dessous de la conscience. À partir des pathologies de Schneider, le philosophe trouve la voix et les gestes d'un corps dont la conscience est fragmentée, un corps qui n'exprime pas sa propre expérience car il n'a pas au-dessus de lui un pouvoir d'organisation auquel il pourrait se soumettre :

Jamais Sch. n'éprouve le besoin de parler, jamais son expérience ne tend vers la parole, jamais elle ne suscite en lui une question, elle ne cesse pas d'avoir cette sorte d'évidence et de suffisance du réel qui étouffe toute interrogation, toute référence au possible, tout étonnement, toute improvisation. On aperçoit par contraste l'essence du langage normal: l'intention de parler ne peut se trouver que dans une expérience ouverte, elle apparaît, comme l'ébullition dans un liquide, lorsque, dans l'épaisseur de l'être, des zones de vide se constituent et se déplacent vers le dehors (Merleau-Ponty, 1945, pp. 228-229).

Or, devant la description de Merleau-Ponty de la maladie en contraste avec l'expérience normale, je m'interroge sur le vrai pouvoir du corps. Que peut-il faire par lui-même? Il se limite à une relation immédiate avec l'extérieur. Son mouvement est dirigé par les nécessités biologiques essentielles. Il réalise seulement les mouvements qui sont nécessaires au maintien de la vie. Les troubles de Schneider empêchent le malade d'accéder à la dimension abstraite de l'existence. Il ne peut pas imaginer ou planifier le futur, ni danser, ni raconter une histoire vraie ou fictionnelle, ni tomber amoureux ou avoir des amis, il n'a pas d'opinions politiques. Il fait seulement ce que son corps permet de faire,

458). Selon Moura, avec qui je suis d'accord, ce passage explicite l'idéalisme de la Phénoménologie de la perception et la présence de la conception sartrienne d'existence chez Merleau-Ponty : « Ora, se ali [dans la « Phénoménologie de la perception »] a análise ainda era dominada pela oposição 'abstrata' entre natureza e criação, era antes de tudo pela prisão do autor à ontologia de St.-Germain-des-Près. Era esse namoro nem um pouco discreto que o levava a contrapor a natureza à 'existência', assim como se opõe uma 'plenitude de ser' inteiramente 'inerte' a uma subjetividade temporalizada que é, antes de tudo, 'não-ser', potência de 'niilizar' que nos habita, pura 'produtividade' em que todo 'sentido' encontrava a sua raiz. E se esse apego à ontologia sartreana é comprometedor, é sobretudo porque, por meio dele, o discurso 'fenomenológico' se fazia herdeiro inconsciente da ontologia cartesiana que, epidermicamente, ele se esforçava em combater » (Moura, 2004, p. 324). Dans le contexte de discussion sur le langage, Le visible et l'invisible reconnait cet idéalisme de la Phénoménologie de la perception et par conséquent la liaison entre sens et cogito présente dans cet ouvrage: « Ce que j'appelle le cogito tacite est impossible. Pour avoir l'idée de 'penser' (dans le sens de la 'pensée de voir et de sentir'), pour faire la 'réduction', pour revenir à l'immanence et à la conscience de... il est nécessaire d'avoir les mots » (Merleau-Ponty, 2004, p. 222). Donc il était impossible de défendre une existence symbolique - capable d'aboutir l'univers du langage - sans l'intervention de la conscience parlée, et non pas seulement tacite, corporelle et anonyme. L'avènement du symbolique est simultané à l'avènement du langage et, par conséquent, du cogito. 
car il a perdu la vision spirituelle du monde. Raison par laquelle il n'a pas de « question » et « son expérience ne tend pas vers la parole ».

La plupart des lecteurs de Merleau-Ponty trouve dans l'analyse de la pathologie la découverte de la normativité corporelle ${ }^{2}$. Merleau-Ponty ne réduit pas l'organisme à une machine, par contre, il met l'accent sur la puissance d'adaptation provenant du corps. Donc il critique la tradition moderne et cartésienne qui consiste à réduire le corps au physique. Ce constat est correct mais je voudrais rappeler qu'au début des années quarante, un autre philosophe a publié un livre important sur le thème de la pathologie. Il s'agit de Canguilhem et de son œuvre « Le normal et le pathologique », où ce philosophe critique aussi le modèle cartésien, ce qui signifie qu'il refuse l'identification entre corps et machine. Il a utilisé les mêmes recherches scientifiques que Merleau-Ponty mais pour arriver à des conclusions plus radicales ${ }^{3}$.

Qu'est-qui explique l'expérience biologique? Selon Canguilhem, il faut remarquer la puissance normative de l'expérience vitale. L'organisme cherche le plaisir et refuse la souffrance et le danger. L'organisme est un pôle normatif qui s'oriente en fonction des obstacles qu'il rencontre dans l'environnement. Il crée les comportements nécessaires pour surmonter les difficultés. De cette manière, il crée son propre monde parce qu'il n'accepte pas les choses telles qu'il les reçoit. On peut dire que l'organisme adapte la nature à ses besoins. Donc, l'organisme n'est pas un pouvoir passif d'adaptation, car il a la puissance créative de transformer les obstacles de la vie en moyens d'existence. Dans le cas de l'humain, Canguilhem explique que la puissance normative vitale donne origine à la technique et à la connaissance. En même temps, elle ouvre la possibilité de la création de normes partagées par les hommes, et permet la vie en société. Il y a un genre de dédoublement de la puissance normative de l'organisme, à tel point que nous pouvons expliquer la culture humaine à partir de cette puissance originelle ${ }^{4}$.

2 Cf. par exemple: "o corpo manifesta uma unidade autônoma, um 'sistema de equivalências', de transponibilidade, que Merleau-Ponty denomina esquema corporal, 'pelo qual as diferentes tarefas motoras são instantaneamente transponíveis. Temos aqui [...] uma unidade inteiramente autônoma, que nada deve a uma consciência, que se passa no corpo considerado apenas como potência motora" (Moutinho, 2006, p. 138). Je pense que cette analyse est vraie quand on se considère le comportement normal. Le malade ne peut pas coordonner l'existence vers le possible, son corps organise l'environnement selon les nécessitées vitales immédiates, et non pas selon la liberté. L'horizon des attitudes symboliques et abstraites est fermé pour Schneider.

3 J'ai présenté une analyse de la différence entre Merleau-Ponty et Canguilhem dans le livre «A Prosa de Dora » (Ramos, 2013, pp. 138 et seq.).

4 Cette réflexion est dédoublée par Canguilhem en 1963-1966, dans la période concernant le deuxième essai publié dans Le normal et le pathologique, les « Nouvelles réflexions concernant le normal et le pathologique (1963-1966) ». Selon Le Blanc, « la science est repensée [...] dans une relation direct à la vie. Ce déplacement de perspective est imputable à deux raisons. Premièrement, la vie n'est plus pensée en 1966 à partir du vital mais à partir du social. Du même coup, ce qui était exclu da la sphère vitale, la science, à titre d'élément 
Dans les années quarante, ce pas radical n'est pas donné par la philosophie de Merleau-Ponty. Pour lui, la sociabilité et toutes les créations humaines exigent l'avènement de la conscience. Car seule la conscience peut établir une relation symbolique avec les choses et avec les autres. Je voudrais m'arrêter un instant sur ce concept. Qu'est-ce que Merleau-Ponty entend par symbolique dans la première phase de sa pensée? Dans « La structure du comportement », il souligne que la réalité perçue par la conscience peut être divisée sous trois formes: la réalité physique, organisée par les lois de la nature; la réalité vitale dans laquelle on trouve les organismes en dialogue avec leur environnement (cette réalité se réalise sous des normes d'adaptation biologique); et la réalité symbolique, c'est-à-dire la réalité humaine, coordonnée par des valeurs et traversée par le langage.

Selon Merleau-Ponty, quelle la différence entre la forme vitale et la forme humaine? Pourquoi la normativité vitale ne peut pas expliquer l'existence humaine ou symbolique?

Pour répondre à ces deux questions, il faut comprendre que Merleau-Ponty a une vision plus étroite de la créativité de l'organisme que celle de Canguilhem. Lorsque Canguilhem souligne l'aspect inventif de la vie (c'est-à-dire qu'il voit dans les comportements vivants une puissance infinie de transformer l'environnement), Merleau-Ponty comprend le pouvoir d'adaptation comme limité. La vie crée ce qu'il faut pour s'adapter et se conserver. Elle est incapable d'aller plus loin que cela. Sur ce point je voudrais rappeler une assertion de Canguilhem quand il dit que la vie se développe dans le risque, et qu'elle cherche à se réaliser comme un luxe ${ }^{5}$. La vie crée plus que le nécessaire. Merleau-Ponty quant à lui n'est pas d'accord avec cette perspective. Il réduit la vie à la résistance précaire au danger, et au désir de se préserver. Le luxe auquel Canguilhem se réfère n'est pas possible dans l'existence biologique. C'est seulement quand un individu dirige son mouvement vers le monde d'une manière désintéressé, que la vie symbolique et abstraite devient possible. Pour que ceci se matérialise, il faut l'avènement de la conscience' .

social, est réintroduit dans la vie, dès lors que le vital est pensé par référence au social. La science, à titre de détermination sociale et anthropologique, est pensée comme forme de la normativité sociale. La science, en tant qu'élément social, contribue à une nouvelle détermination de la vie entreprise dans les Nouvelles Réflexions. Deuxièmement, les concepts de la biologie moderne, sens, information, erreur..., apparaissent comme des structurations internes du vivant. La science produit ainsi une nouvelle allure de la vie. La connaissance est pensée comme une stratégie vitale au même titre que la technique » (Le Blanc, 1998, p. 109).

5 « Être en bonne santé c'est pouvoir tomber malade et s'en relever, c'est un luxe biologique » (Canguilhem, 2007, p. 132). Ce passage peut être trouvé dans la deuxième édition du livre " Le normal et le pathologique », mais cette perspective optimiste concernant le pouvoir du vivant est toujours présente chez Canguilhem car les comportements anormaux sont l'expression d'une capacité d'invention ouverte par le vivant.

6 La distinction entre la parole parlante et la parole parlée est cruciale ici. Elle montre que l'ouverture au possible et par conséquent la liberté est quelque chose qui dépasse la vie: « on pourrait distinguer une parole parlante 
Dans la «Phénoménologie de la perception», Merleau-Ponty montre que la conscience de soi est simultanée par rapport à la conscience des objets. Le moi se découvre dans sa relation avec le monde, et dessine son projet de vie. À chaque moment, il est projeté dans le futur où les possibilités de réalisations sont toujours ouvertes. Il n'est pas déterminé par les conditions données au présent, car le futur est son œuvre continuelle. Avec la conscience dans sa forme essentielle qui est le temps, l'histoire humaine surgit. Cette description nous permet de voir l'abîme entre la vie biologique et la vie consciente chez Merleau-Ponty. La première (leben) ne peut pas aller au-delà de sa propre conservation. Elle résiste au danger extérieur qui la menace et se reproduit pour préserver son espèce. La vie consciente (erleben) au contraire affronte le danger car le monde avec ses horizons infinis contient plusieurs possibilités de création. La vie humaine n'est pas reproduction et préservation, mais création et invention. Elle est toujours au-delà d'elle-même.

Après les considérations présentées ci-dessus, je voudrais revenir à la philosophie de la vie selon Canguilhem. J'ai dit que Canguilhem insiste sur la puissance originelle de l'organisme. La polarisation normative donne sens au monde extérieur car elle dessine les objets que l'organisme poursuit et ceux qu'il évite. Sauf en cas de comportement catastrophique, même un organisme malade conserve un peu de cette puissance: il n'a plus la force suffisante pour vaincre immédiatement les grandes menaces extérieures, mais il structure une nouvelle relation avec l'extérieur capable de lui fournir une existence stable jusqu'au dépassement de cette difficulté. Le comportement anormal ouvre la possibilité de création d'une nouvelle normativité. Donc l'organisme peut tomber malade pour affronter les menaces et pour élargir ses domaines. Or, le pouvoir de vaincre les dangers et d'aller au-delà d'elle-même est essentiel à la vie. La vie humaine est le dédoublement de ce pouvoir. Donc, pour Canguilhem, il n'y a pas d'abîme qui séparerait la vie biologique de la vie humaine.

Qu'est-ce qui empêche Merleau-Ponty de suivre un chemin théorique semblable? La réponse est évidente: c'est un phénoménologue. Même s'il cherche à construire une phénoménologie de la perception, il a besoin d'une

et une parole parlé. La première parole est celle dans laquelle l'intention significative se trouve à l'état naissant. Ici l'existence se polarise dans un certain 'sens' qui ne peut être défini par aucun objet naturel, c'est au-delà de l'être qu'elle cherche à se rejoindre et c'est pourquoi elle crée la parole comme appui empirique de son non-être. La parole est l'excès de notre existence sur l'être naturel » (Merleau-Ponty, 1945, p. 229). Aussi la sexualité met en jeu la relation d'expression entre la vie biologique et la vie personnelle, de sorte que l'on remet en question l'humanité du malade : «Ainsi la vue, l'ouïe, la sexualité, le corps ne sont pas seulement les points de passage, les instruments ou les manifestations de l'existence personnelle: elle reprend et recueille en elle leur existence donnée et anonyme » (Idem, p. 186). Cet acte de reprendre le donné et de surmonter les nécessités vitales est impossible à la fois dans la vie anonyme et dans la maladie. 
conscience pour soutenir la vision du monde. Malgré l'importance de l'expérience corporelle dans ses premiers livres, l'organisation de cette expérience dépend de la conscience. Donc, la philosophie de la perception est une nouvelle sorte d'idéalisme, même si elle est attachée à l'expérience sensible. Comment MerleauPonty peut-il échapper à ce piège, c'est ce que nous allons présenter. Je propose une stratégie: explorer la théorie de l'imaginaire chez Merleau-Ponty.

\section{2}

La conception de l'imaginaire présentée dans la «Phénoménologie de la perception » est très proche des idées défendues par Sartre dans « L'Imaginaire ». Merleau-Ponty différencie perception et imagination en affirmant que la première a une cohésion plus forte que la seconde. Dans la perception, le sujet trouve une séquence d'apparitions cohérentes qui peut être explorée indéfiniment. Dans l'imagination, au contraire, les apparitions sont créées par la conscience qui explore son propre pouvoir d'inventer un espace fictionnel dont le siège est le corps (c'est la différence entre Merleau-Ponty et Sartre, la présence du corps). À partir de cette description, Merleau-Ponty oppose la profondeur de la perception à l'aspect superficiel de l'imagination. Il y a deux moments dans la Phénoménologie de la perception où le philosophe explicite cette dualité : quand il analyse la schizophrénie (Merleau-Ponty, 1945, pp. 385 et seq.) et quand il aborde les rêves (Merleau-Ponty, 1945, p. 437).

Les schizophrènes vivent dans un monde imaginaire. Ce monde a deux aspects : il est construit par des expériences ponctuelles (éclatements et piqûres, par exemple), et il est tout à fait solitaire. Bien qu'il soit vécu comme extérieur et partageable, le monde imaginaire du schizophrène, par sa propre fragilité, doit être préservé de la communication.

Cependant, quel est le moment privilégié dans l'expérience normale où l'imagination est au centre ? L'expérience onirique est le domaine de l'imaginaire. Pendant le rêve, la conscience s'abandonne à la prolifération des images. Il y a là un nouveau défi interprétatif, car il faut expliquer le sens des images qui surgissent dans le rêve. Depuis Freud, l'incohérence de l'imaginaire onirique peut être interprétée à partir de la conjonction entre les images quotidiennes du rêveur, et le désir qu'il poursuit mais ne peut pas assumer. Freud a découvert deux textes qui se superposent dans l'imaginaire onirique : le sens manifeste et le sens latent. Le premier est simplement la figuration d'un désir plus profond que l'analyste devra interpréter à partir des images données. C'est le schéma freudien classique, c'est-à-dire que l'analyste a la mission d'établir un jeu symbolique entre les images qui sont présentes dans le rêve, et le désir qui les 
motive. Au fond, l'objectif est de traduire d'une manière claire et consciente un désir inconscient qui est soumis à la censure.

Merleau-Ponty utilise ce schéma, mais nous savons qu'il était un lecteur de Politzer. Donc le philosophe critique la conception freudienne de l'inconscient. Pour Merleau-Ponty l'inconscient n'est pas un ensemble d'entités réelles qui existeraient derrière la conscience. Comme Politzer (2003), il refuse toute conception réaliste. Mais, si les rêves ne sont pas la figuration d'un désir réel refoulé, un inconscient réifié, comment la philosophie peut-elle les expliquer? Ici, la superficialité de l'imaginaire explique la diffusion d'un désir qui se propage sans obstacle. Pendant le rêve, le sujet ne perçoit rien: il n'a pas de contact avec les choses du monde, mais seulement avec les images qui sont créées par lui-même. Son désir est donc libéré de la résistance de la réalité. Merleau-Ponty pense que le rêveur est un désir pur qui ne cherche que la satisfaction immédiate. Par conséquent, Merleau-Ponty défend que les images oniriques n'ont pas un sens sexuel, car il n'y a pas d'autre sens dans les rêves. Pour trouver un sens sexuel, il faudrait trouver encore un autre sens dans le rêve, et le détacher des images qui seraient sexuelles. Mais selon Merleau-Ponty, l'imaginaire onirique n'a pas de fond, il est une surface homogène, un genre d'écran où le désir dessine son intentionnalité7.

À partir des exemples que j'ai étudiés jusqu'au présent, je peux observer le caractère essentiel de l'imaginaire, c'est-à-dire sa superficialité. Ici, il n'y a pas de jeu entre figure et fond, entre les dimensions du temps ou entre manifeste et latent. C'est la conscience perceptive et éveillée qui produit ces jeux. Donc, dans ces deux cas, schizophrénie et rêve, l'imaginaire est immédiat, et il ne comporte pas de dimension symbolique ${ }^{8}$. Un autre aspect que je peux aussi explorer est celui du rôle du corps dans cette expérience. Selon Merleau-Ponty, toutes les images qui sont figurées dans l'univers onirique sont fournies par la sensibilité corporelle. Le corps fournit les structures de la sensibilité qui rendent l'imagination possible. Donc, selon Merleau-Ponty, nous pouvons dire que le support de l'imaginaire, dans les deux cas, est le corps, et non pas la conscience. Par conséquent, l'imagination onirique, comme la schizophrène, montre le pouvoir du corps. Elles révèlent qu'il y a une créativité corporelle (permise par

7 « L'incendie qui figure dans le rêve n'est pas pour le rêveur une manière de déguiser sous un symbole acceptable une pulsion sexuelle, c'est pour l'homme éveillé qu'il devient un symbole ; dans le langage du rêve, l'incendie est l'emblème de la pulsion sexuelle parce que le rêveur, détaché du monde physique et du contexte rigoureux de la vie éveillé, n'emploie les images qu'à raison de leur valeur affective » (Merleau-Ponty, 1945, p. 437).

8 II y a d'autres exemples explorés par Merleau-Ponty dans la « Phénoménologie de la perception ». La possibilité de assumer un rôle - être un acteur - ou la capacité de raconter une histoire fictionnelle sont décrits comme activités qui exigent en même temps l'imagination et la référence au possible ou l'absent. Donc, ils sont des activités propres de l'expérience symbolique. Les malades étudiés par Merleau-Ponty sont incapables d'accomplir ces activités. Le rêve par contre est toujours pensé comme superficiel et immédiat dans ce livre. 
les structures sensibles), mais par contre, elle en dévoile les limites. Ce que le corps produit n'est jamais traversé d'une énergie symbolique. Tout ce qui est produit à partir du corps est marqué par le caractère immédiat et direct, et donc ne comporte pas le mystère, la profondeur et l'ouverture du symbolique. Cette ouverture est produite par la conscience personnelle qui recueille cette expérience du corps anonyme.

Chez Merleau-Ponty, tout au moins pendant les années quarante, le pathologique et l'imaginaire sont le domaine de ce que Canguilhem a nommé la normativité vitale. Cependant, contrairement à Canguilhem, Merleau-Ponty ne croit pas que la normativité vitale ait le pouvoir de faire naître la culture humaine. Donc, les productions du corps ont besoin d'être sublimées par la conscience présente, pour devenir symboliques. La culture humaine dépend de cette transformation pour se réaliser. Ainsi, une transformation de cette perspective selon laquelle la culture est associée à l'avènement de la conscience dépend d'une nouvelle idée du symbolique et de la vie. Je crois que les nouvelles études merleau-pontiennes de cas décrits par Freud et l'investigation de la biologie contemporaine sur les comportements vitales fournissent dans les années cinquante les conditions de cette transformation. Il faut que Merleau-Ponty découvre la puissance symbolique de la vie et la profondeur de l'imaginaire onirique pour qu'une compréhension élargie de la production symbolique soit possible.

\section{3}

Dans le cours sur la notion de Nature (1957-1958), Merleau-Ponty analyse trois caractères essentiels à la vie biologique. Le premier aspect provient de l'embryologie. La science montre que l'organisme en formation porte en puissance des états qui seront développés ultérieurement, quant il arrive à la maturité. Cela révèle que l'organisme ne se réduit pas à ses pouvoirs actuels, car il anticipe à chaque geste les comportements qu'il sera capable d'aboutir dans le futur. Donc, il y a une temporalité dense inscrite dans le développement de l'organisme'.

Le second aspect concerne la relation avec l'environnement, et MerleauPonty suggère la possibilité de refuser l'adaptation comme la seule perspective

9 L'organisme est décrit d'après la notion de totalité. Mais la principale mutation ici est l'idée selon laquelle l'organisme est ouvert sur un avenir; donc son comportement n'est pas orienté par les besoins immédiats: "Le corps vivant est en puissance de se transformer soi-même; c'est un corps qui constitutivement bascule vers l'avenir. Les analyses de Coghill [Anatomy and the problem of behaviour] nous orientent vers ce qu'il faudrait appeler une 'possibilisation' du corps vivant » (Bimbenet, 2004, p. 251). Selon Merleau-Ponty, « II y a du possible dans l'organisme. L'embryon n'est pas simple matière, mais matière qui fait référence à l'avenir » (Merleau-Ponty, 1994, p. 193). 
possible pour comprendre l'ouverture de l'organisme sur l'extérieur. La biologie contemporaine défend l'idée que l'instinct n'a pas d'objets définis par nature. Au contraire, l'instinct est fondamentalement sans objet (objektlos) $)^{10}$. Il y a un schéma prédéfini selon lequel l'organisme cherche quelque chose à l'extérieur. Cela signifie que l'organisme est ouvert aux stimuli extérieurs, mais il n'est pas conditionné à chercher un objet précis. Merleau-Ponty dit que l'organisme flotte sur les images qu'il trouve, mais il ne s'arrête pas face à un objet. Par conséquent, nous pouvons dire que l'instinct se définit par un désir qui ne peut pas être satisfait, car il ne prescrit pas ce qu'il cherche. L'adaptation exigerait une relation plus apaisée avec l'extérieur que celle produite par un désir insatiable. Quand la biologie met l'énergie pulsionnelle au centre de l'organisme, elle montre que l'adaptation ne ferme pas l'horizon du comportement vital. La vigueur du désir est toujours présente et peut créer de nouvelles configurations de comportement. En d'autres mots, la vie produit plus que le nécessaire à l'adaptation immédiate car le désir la condamne à être toujours au-delà d'elle-même.

Le troisième aspect concerne la question du mimétisme. Dans ce cas, la biologie contemporaine fournit des éléments responsables du refus de la conception utilitariste de la production visuelle dans l'univers biologique. Le biologiste Lorenz raconte une expérience très simple au cours de laquelle l'usage du corps animal est tout-à-fait gratuit, et donc n'exprime pas une finalité immédiate d'adaptation. Depuis longtemps la biologie voit dans la ressemblance entre l'organisme et l'extérieur une preuve de sa puissance d'adaptation. Par exemple, le fait qu'un papillon imite les couleurs de l'arbre où il vit pourrait signifier qu'il dispose d'un mécanisme de défense. Lorenz rappelle que les musées d'histoire naturelle sont pleins de représentations dans lesquelles ces mécanismes sont interprétés de cette manière. Mais quand le biologiste observe le phénomène dans la nature, il perçoit que le papillon reste la plupart du temps les ailes fermées. Donc le mécanisme se montre peu efficace du point de vue du camouflage. Il faut trouver un sens différent de l'utilitaire pour expliquer le mimétisme. Merleau-Ponty préfère l'expliquer par l'exubérance de la vie. Parfois, l'aspect visible de l'animal peut l'aider à échapper à un ennemi, mais cette possibilité ne suffit pas à expliquer pourquoi la visibilité est si complexe

10 Merleau-Ponty fait référence à l'univers onirique. Mais cet univers n'est pas immédiat. Au contraire, il y a une densité temporale et symbolique qu'opère ici : "L'instinct est une activité instaurée du dedans, mais qui possède une cécité, et qui ne connait pas son objet. C'est ainsi que l'étourneau, sans jamais avoir présenté un tel comportement ni l'avoir jamais vu chez un congénère, présente tout le développement de la chasse aux mouches, bien qu'il n'y ait absolument aucune mouche dans son entourage. [...] Cet instinct ne s'accomplit pas en vue d'une fin, c'est une activité pour plaisir. [...] Ainsi une sorte de référence à l'inactuel, de vie onirique, se manifeste dans ces activités instinctives à l'état pur. Si ces actes se produisent la plupart du temps par référence à un objet, ils sont tout autre chose que la référence à un objet, la manifestation d'un certain style » (Merleau-Ponty, 1994, p. 251). 
dans les phénomènes biologiques. L'apparence animale se développe d'une manière excessive et gratuite, et ses effets sont parfois imprévisibles. Par exemple, beaucoup d'activités mimétiques sont reconnues pour leur pouvoir de communication. À nouveau, dans ce « développement de l'instinct en fonction symbolique » (Merleau-Ponty, 1994, p. 256), le philosophe découvre un excès positif qui projette la vie au-delà d'elle-même.

Les trois caractères essentiels à la vie que Merleau-Ponty a étudiés dans le cours sur la Nature bouleversent la conception de la vie qu'il avait développée dans ses premiers livres. À travers la biologie contemporaine, le philosophe dévoile la temporalité essentielle du vivant. Cela exige le refus du paradigme de l'adaptation, lequel devrait être remplacé par une idée plus complexe et donc élargie de la vie. En d'autres termes, l'être vivant porte la puissance de la communication et le projet d'un futur indéfini. Pour Merleau-Ponty, le comportement de l'organisme anticipe ce que la vie humaine va développer d'une manière plus intense: la culture ${ }^{11}$. Le cours sur la Nature efface la frontière qui séparait l'homme des animaux et remet en question l'aspect fondamental qui permettait de soutenir la différence entre vie et expression symbolique. Par conséquent, il fournit une pièce qui permettra de construire un nouvel édifice conceptuel où la production symbolique trouvera sa source dans la vie elle-même. En même temps, Merleau-Ponty devra retourner à l'étude de l'imaginaire (dans lequel la vie maîtrise plus que la conscience), pour décrire le symbolisme particulier de l'hystérie. Le philosophe trouve dans l'expérience hystérique la naissance d'une production symbolique qu'il caractérise comme barbare ou sauvage.

Qu'est-ce que cela signifie ? Ici, les références proviennent de la psychanalyse et de la pensée de Politzer ${ }^{12}$. Fondamentalement, ces références conduisent à une

11 «Ce que montre l'animal, ce n'est pas une utilité ; son apparence manifeste plutôt quelque chose qui ressemble à notre vie onirique. Sans doute, en un certain sens, le cérémonial sexuel est-il utile, mais il n'est pas utile que parce que l'animal est ce qu'il est. Une fois qu'ils sont, ces manifestations ont un sens, mais le fait qu'elles soient telles ou telles n'aucun sens. De même qu'on peut dire de toute culture qu'elle est à la fois absurde et qu'elle est berceau du sens, de même toute structure repose sur une valeur gratuite, sur une complication inutile » (Merleau-Ponty, 1994, p. 246). À ce propos, Bimbenet écrit : " Définie comme mouvement, être en puissance reconduit dans son actualisation même, la perception vivante nous apparait en effet comme le fait d'un organisme qui est manque avant d'être plénitude, et qui ne peut pas se chercher qu'en dehors de luimême. Or c'est exactement cette négativité qui vient s'illimiter dans le bâti charnel du corps humain. Comme recherche du dedans dans le dehors et du dehors dans le dedans, la chair s'obsède d'une totalité qui, comme l'interrogation vitale, puise aux seules ressources du visible » (Bimbenet, 2004, p. 271).

12 Selon Saint-Aubert : 'L'intégration' merleau-pontienne de la psychanalyse est en dialogue avec d'autres tentatives contemporaines, notamment celle de Scheler, les 'psychanalyses' de Bachelard, et, surtout, la 'psychanalyse existentielle' de Sartre. C'est d'abord contre celle-ci que ses derniers écrits évoquent le projet d'une 'psychanalyse ontologique'. Latente dès le chapitre de la Phénoménologie de la perception sur le corps comme être sexué, cette opposition éclate dans les inédits de la fin des années quarante, au cœur même de la naissance du concept de chair, et sera développée dans les plusieurs manuscrits plus tardifs, à commencer par le cours sur la passivité de 1955 »(Saint-Aubert, 2006, p. 11). 
expression qui n'est pas configurée par la conscience. Au contraire, son aspect sauvage est dû aux équivalences qui prolifèrent dans la relation désirante, et essentiellement sensible. La conscience n'est plus l'origine du symbolique, car la production symbolique est ancrée dans l'expérience vécue par un corps désirant et expressif. Pour interpréter la fécondité que l'hystérique peut exprimer par les mots, les gestes et les images, le philosophe a besoin d'une herméneutique. Merleau-Ponty cherche, dans le cours sur la passivité, l'herméneutique qui permet d'interpréter les images et les mots utilisés dans ce cas. Du point de vue philosophique, Merleau-Ponty affronte deux défis: produire une théorie de l'imaginaire qui comporte l'élucidation du désir inconscient et une théorie du langage capable de traduire les symptômes vécus par des signes partageables.

\section{4}

Freud a affirmé que le rêve est la réalisation d'un désir refoulé. Le travail de l'analyste consiste à libérer le désir de la censure, c'est-à-dire faire transiter l'énergie pulsionnelle de l'espace inconscient vers la conscience. Il y a ici une conception matérialiste du psychisme selon laquelle l'inconscient est une espèce de maison du désir. D'après les observations ci-dessus, selon Merleau-Ponty, la réalité de l'inconscient est quelque chose d'obscur et d'abstrait. L'énergie pulsionnelle n'est pas une chose réelle, au contraire, c'est une manière de se comporter ou d'être au monde. Autrement dit, il n'y a pas un phénomène strictement pulsionnel, car le désir convoque l'existence toute entière pour s'exprimer et se réaliser. La question « où est le désir? » n'a pas de sens, car le désir n'est pas une chose qui pourrait se loger dans un espace défini, conscient ou inconscient. Le désir concerne le mouvement de l'existence, et sa définition doit le considérer comme une intentionnalité et pas comme une substance.

Cette considération fournit le contexte pour un projet herméneutique capable d'expliquer les images oniriques qui apparaissent dans les rêves. La première considération à ce sujet concerne la force de ces images. Comment expliquer qu'un rêve puisse impressionner plus qu'une perception éveillée? La théorie classique de l'imaginaire défend que la fantasmagorie du rêve n'ait pas une profondeur comparable à celle de la perception. À travers la perception, le monde nous est donné sous ses divers aspects. En ce sens, la perception a devant elle un horizon infini. L'imaginaire par contre n'a pas de consistance, c'est une surface qui peut être détruite par la raison ou par une perception réelle. Or, si cette conception était correcte nous aurions des difficultés pour expliquer la force du rêve. Comment quelque chose de si fragile et superficielle peut être si perturbante? Il est vrai que la force de l'imaginaire ne réside pas dans 
la réalité des choses qu'il produit. Cette force capable de troubler l'homme le plus raisonnable trouve son explication dans la densité symbolique qu'il porte.

Les images du rêve ne se réduisent pas à ce qu'elles montrent. Le rêve est un message qui se dirige vers les objets du désir. Mais il faut considérer que les objets du désir ne sont pas clairs. Quand l'analyste étudie les représentations de l'hystérique, il perçoit qu'une même figure représente plusieurs objets. Par exemple, la figure du médecin comporte le geste du père et la voix d'un ami perdu. Cette ambiguïté fournit la clef qui donne la possibilité de pénétrer dans le mystère de l'univers onirique. Il ne faut pas donner réalité à l'imaginaire pour comprendre sa force. Il est suffit d'explorer sa capacité essentielle de faire référence à plusieurs d'objets à travers une image. Dans le rêve, l'image est un pivot autour duquel les objets désirés circulent. Donc, l'herméneutique des images devrait explorer l'ambiguïté particulière de chaque image qui apparait dans les rêves. En d'autres termes, le désir (comme l'instinct animal) est sans objet définit.

\section{5}

Pour comprendre la parole de l'hystérique, Merleau-Ponty élabore une théorie semblable à celle que nous avons développée ci-dessus. Les mots qui racontent son histoire ou encore qui décrivent ses rêves, portent une sorte d'ambiguïté pleine de sens. Dans ce cas encore, le philosophe ne trouve pas, dans la confusion apparente du récit, l'indice d'un défaut. Par contre, ce qui brouille les idées est un excès de référentiel. Quand un mot est dit, il évoque un sens immédiat, mais aussi beaucoup de références qui gravitent autour de lui. Le récit engendre un paysage dans lequel les épisodes du passé, le contexte présent et le projet futur cohabitent. Comme dans le cas des images, les mots portent une densité symbolique qui s'exprime par l'ambiguïté qui les traverse.

Ainsi, l'imagination et le langage de Dora dépassent le moment présent et l'intérêt immédiat. Ce dépassement n'est pas accompli par la conscience, au contraire, il anime l'imaginaire et la parole spontanée de l'hystérique. Il est inscrit dans la manière d'être de l'hystérique, dans son style. Il faut abandonner ou tout au moins diminuer le pouvoir de maîtrise de la conscience, pour libérer la créativité qui subsiste sous l'existence personnelle. En définitive, la vie corporelle n'est pas la révélation d'une normativité vitale attachée au immédiat et limité par la nécessité. Au contraire, elle est une usine symbolique où les dimensions du temps et les dimensions de l'espace cohabitent étroitement sous le régime de la promiscuité ${ }^{13}$. 
Concernant l'imagination de Dora, et l'aspect symbolique des images que se situent dans tout les dimensions du temps, le présent aussi bien que le passé et le futur, Merleau-Ponty montre que l'imagination hystérique est capable de récupérer les expériences traumatiques du passé et d'ouvrir un nouveau futur. Le «cas Dora» explicite que l'hystérique n'est pas complètement soumise à l'immédiat, car son imagination dépasse la frontière du présent et touche le passé comme le futur. Ce dépassement n'est pas réalisé par la conscience, mais possibilité par la sensibilité corporelle qui s'exprime dans les rêves, gestes et parole hystériques. Ce qui est explicite ici est un nouvel horizon théorique capable d'articuler l'ontologie et l'esthétique. Après tout, l'imagination de l'hystérique et son récit sont capables, selon Merleau-Ponty, d'apporter à la visibilité et à l'expression notre rapport charnel avec l'être. Ce qui est invisible à l`expérience normale devient imaginaire et récit par l'expérience hystérique ${ }^{14}$.

\section{6}

La «Phénoménologie de la perception » décrit l'expérience du corps propre à partir de la vision du cogito. Par conséquent, cette expérience est soumise aux exigences et aux formalisations de la conscience. En d'autres termes, elle n'est pas présentée par elle-même, car le corps apparaît selon la perspective que la conscience actuelle fournit de lui. Pour expliciter cet idéalisme, j'ai analysé la principale voix donnée au corps au début de la pensée de Merleau-Ponty: la voix pathologique. Selon le philosophe, quand avons-nous la chance d'écouter et d'entendre le pouvoir du corps? Quand il tombe malade. Les troubles de Schneider décrits dans la «Phénoménologie de la perception » rendent explicite l'impuissance symbolique du comportement pathologique et, même si MerleauPonty n'avait pas cet objectif supplémentaire, ils montrent que cette impuissance est vraiment l'impuissance du corps propre. Le sujet malade est un être soumis à la normativité immédiate de la vie biologique, raison par laquelle il peut découvrir à chaque moment la finitude et la fragilité de l'existence:

14 Selon « L'institution et la passivité » « Une métaphysique de la sexualité est nécessaire : il est nécessaire de comprendre pourquoi et comment, en vertu de sa structure propre, elle rend possible une Leistung qui intéresse le tout de la vie de l'individu. La sexualité même, n'étant pas une 'fonction naturelle', a besoin d'être expliquée. Le problème qu'elle pose (souci de le résoudre) est de savoir comment une vie peut entrer dans autre vie ou celle-ci la recevoir. L'amour physique n'est le symbole universel, n'est intéressé dans toute opération humaine et n'y apporte sa contribution, son poids, son volant, que parce qu'il symbolise lui-même au plus haut point l'incarnation, la corporéité, le rapport charnel avec autrui. La psychanalyse est bien, en dernière analyse, psychanalyse existentielle, mais non pas au sens de Sartre, c'est-à-dire comme révélation d'une position prise par 'la liberté', mais comme révélation de l'intercorporéité, du montage Ego-autrui, tel qu'il est réalisé par chacun, du système symbolique installé dans notre machine à vivre » (Merleau-Ponty, 2003, p. 246). 
Si le corps peut symboliser l'existence, c'est qu'il la réalise et qu'il en est l'actualité. Il seconde son double mouvement de systole et de diastole. D'une part, en effet, il est la possibilité pour mon existence de se démettre d'elle-même, de se faire anonyme et passive, de se fixer dans une scolastique. Chez la malade ${ }^{15}$ dont nous parlions, le mouvement vers le futur, vers le présent vivant ou vers le passe, le pouvoir d'apprendre, de murir, d'entrer en communication avec autrui se sont comme bloqués dans un symptôme corporel, l'existence s'est nouée, le corps est devenu 'le cachette de la vie'. Pour le malade, il n'arrive plus rien, rien ne prend sens et forme dans sa vie - ou plus exactement il n'arrive que des 'maintenant' toujours semblables, la vie reflue sur ellemême et l'histoire se dissout dans le temps naturel (Merleau-Ponty, 1945, pp. 191-192).

Cela m'a amené à m'interroger sur la temporalité et l'intersubjectivité caractéristique de l'imaginaire. Quel est le temps de l'imaginaire normal? Le futur: il construit un monde intentionnel qui n'existe pas, mais qui est possible et partageable. Quelles sont par contre les caractéristiques de l'imaginaire du malade? Pour répondre à cette question, il y a chez Merleau-Ponty deux exemples «négatifs»: Schneider (il ne peut rien imaginer parce qu'il vit dans le temps présent) et le schizophrène (qui a une imagination solitaire). Or, si on évoque par contre les notes de cours au Collège de France, de 1954-1955, sur la passivité, et les cours sur la Nature, de 1957-1958, on perçoit que Merleau-Ponty examine la vie et l'imaginaire à partir d'une perspective tout à fait différente. La vie perçue dans le comportement de l'organisme montre que l'animal anticipe ce que la vie humaine va développer d'une manière plus intense. La lecture qu'il a faite du « cas Dora » à son tour montre que le philosophe trouve dans l'imaginaire et parole de l'hystérique une expérience symbolique très particulière. Cette expérience exige la compréhension de la puissance symbolique du corps propre et le refus de la position idéaliste assumée par la «Phénoménologie de la perception ». Je trouve dans cette position critique la possibilité de décrire la voix du corps tout en pouvant narrer l'expérience de l'être. Cela indique un tournant de la pensée de Merleau-Ponty vers le corps et la vie. L'art de narrer de l'hystérique, loin d'être un défaut, exige une nouvelle herméneutique capable de comprendre l'articulation symbolique entre passé, présent et futur responsable pour soutenir le sens que traverse les images et les mots évoqués par la narratrice. Depuis les années cinquante, le philosophe tente de rendre compte de une nouvelle compréhension du symbolique pour explorer cette nouvelle possibilité de raconter l'expérience d'être vivant. 


\section{Références}

BARBARAS, R. "Introduction à une phénoménologie de la vie”. Paris: Vrin, 2008. BIMBENET, E. "Nature et humanité. Le problème anthropologique dans l'œuvre de Merleau-Ponty". Paris: Vrin, 2004.

CANGUILHEM, G. "Le normal et le pathologique". Paris: PUF, 2007.

COLONNA, F. "Merleau-Ponty penseur de l'imaginaire". Quiasmi 5, 2003.

LE BLANC, G. "Canguilhem et les normes". Paris: PUF, 1998.

MERLEAU-PONTY, M. "L'institution la passivité. Notes de cours au Collège de France - 1954-1955". Paris: Belin, 2003.

. "La Nature. Cours du Collège de France". Paris: Seuil, 1994.

. "Parcours deux (1951-1961)". Paris: Verdier, 2000.

. "Phénoménologie de la perception". Paris: Gallimard, 1945.

. "La prose du monde". Paris: Gallimard, 2004.

. "Signes". Paris: Gallimard, 2003.

. "La structure du comportement". Paris: PUF/Quadrige, 2001.

. "Le visible et l'invisible". Paris: Gallimard, 2004.

MOURA, C. A. R. "Racionalidade e crise. Estudos de História da Filosofia Moderna e Contemporânea". São Paulo: Discurso, 2004.

MOUTINHO, L. D. S. "Razão e experiência: ensaio sobre Merleau-Ponty”. São Paulo: Ed. Unesp, 2006.

POLITZER, G. "Critique des fondements de la psychologie". Paris: Quadrige/PUF, 2003. RAMOS, S. S. "A prosa de Dora. Uma leitura da articulação entre natureza e cultura na filosofia de Merleau-Ponty”. São Paulo: Edusp, 2013.

RENAULT, A. "Phénoménologie de l'imaginaire et imaginaire de la phénoménologie: Merleau-Ponty lecteur de Sartre et Freud". In: Chiasmi International 5, 2003.

RODRIGO, P. "A La frontière du désir: la dimension de la libido chez Merleau-Ponty". In: Cariou, Barbaras et Bimbenet (orgs.). Merleau-Ponty aux frontières de l'invisible. Milano: Mimesis, 2003.

SAINT-AUBERT, E. "Du lien des êtres aux éléments de l'Être. Merleau-Ponty au tournant des années 1945-1951”. Paris: Vrin, 2004.

. "La 'promiscuité'. Merleau-Ponty à la recherche d'une psychanalyse ontologique". In: Archives de Philosophie, Printemps, 2006. 